九州大学学術情報リポジトリ

Kyushu University Institutional Repository

\title{
AN ORDER RELATION AND FUNDAMENTAL OPERATIONS ON FUZZY NUMBERS AND THEIR APPLICATIONS TO FUZZY LINEAR PROGRAMMING
}

Furukawa, Nagata

Department of Mathematics, Faculty of Science, Kyushu University

https://doi.org/10.5109/13429

出版情報: Bulletin of informatics and cybernetics. 25 (3/4), pp.161-179, 1993-03. Research Association of Statistical Sciences

バージョン:

権利関係 : 


\title{
AN ORDER RELATION AND FUNDAMENTAL OPERATIONS ON FUZZY NUMBERS AND THEIR APPLICATIONS TO FUZZY LINEAR PROGRAMMING
}

By

\author{
Nagata FuruKaWA*
}

\begin{abstract}
An order relation, which satisfies the axioms of a partial ordering, is defined on the set of general fuzzy numbers. An L fuzzy number is defined as a fuzzy number specialized to a certain extent, and fundamental linear operations are introduced on the set of the L fuzzy numbers. After that, characterization theorems of the order among the L fuzzy numbers is given in terms of the parameters of the fuzzy numbers, and it is investigated, on the basis of the theorems, that how the order relation is adapted for the fundamental operations.

Finally, some of these results are applied to two types of fuzzy linear programming problems, in which the coefficients of constraint functions are L fuzzy numbers, to show that both types of the problems can be reduced to nonfuzzy problems. One type of them is reduced to a usual linear programming problem, and another type generates a nonfuzzy new programming problem.
\end{abstract}

\section{Introduction}

Linear programming problems with fuzzy constraints and fuzzy goals have been studied by many authors on the basis of the Bellman-Zadeh principle since the publish of the paper [1]. This principle is used dominatively, especially when the coefficients of the constraint and objective functions are all crisp numbers.

In the case where the linear program involves fuzzy data, for instance, the case where the coefficients of the functions in the program are fuzzy numbers, one has two problems to be solved in advance. The first is to define fundamental operations on fuzzy numbers, and the second is to clarify what an inequality sign between two fuzzy numbers does mean. Dubois and Prade [3] has given the definitions of operations on fuzzy numbers by the use of an extension principle (a fuzzification principle). For the second problem, Dubois and Prade [5] has proposed four indices to describe the relative locations of two fuzzy numbers, and Prade [7] discussed the indices for the general fuzzy sets.

As to the studies of linear programming with fuzzy data, we can refer to the paper [2] by Dubois which is based on the operations and indices in the above literatures.

\footnotetext{
* Department of Mathematics, Faculty of Science, Kyushu University, Fukuoka, Japan
} 
Inuiguchi and Ichihashi [6] discussed the fuzzy linear program by using the concepts of relative modalities improved from the indices in [7]. Tanaka and Asai [8] defined a fuzzy inequality between two fuzzy numbers in another way and discussed the fuzzy linear program related to the inequalities of their meaning. In Dubois and Prade [4], the indices of [5] and [7] are not used and an inclusion sign between fuzzy numbers are adopted in place of the inequality sign.

The fundamental operations on fuzzy numbers defined by Dubois and Prade in [3] are those induced from a nonlinear operation called "an extension principle". Consequently their operations, in general, are not linear operations. There may be little obstacle in applying their operations to various types of fuzzy linear programming as seen in [2], [6], [7] and [8]. However, for the sake of application to nonlinear fuzzy programming, their operations are not suitable on account of the fact that the space of fuzzy numbers does not form a linear space under the operations.

In this paper we first give a new concept of an order relation among fuzzy numbers. The order relation of our definition is a natural extension of the order over real numbers to the set of fuzzy numbers, and satifies the axioms of partial ordering. Next we give a definition of an $L$ fuzzy number, and introduce a new fundamental operations on the set of $L$ fuzzy numbers. Our operations satisfy the linearity unlike the operations of Dubois and Prade.

The final object of this paper is to apply our order relations and our operations to fuzzy linear programming, which has $L$ fuzzy numbers as the coefficients of the constraint and objective functions. In this programming we adopt the order relation defined in this paper in place of the inequality sign. Under these settings we show that our fuzzy linear programming problems can be reduced to nonfuzzy programming problems. In a certain case the problem is reduced to a usual linear programming problem, and in another case it generates a new type of optimization problem. The authors in [2], [6] and [8] have already shown similar results, but their results depend on the level value (or the cut value) chosen to the fuzzy numbers. In contrast with them, in our case, the reduced nonfuzzy programming problem does not depend on any cause such as the level or cut valules.

\section{An Order Relation of Fuzzy Numbers}

Definition 2.1. A real fuzzy number $A$ is defined as any fuzzy set on the space of real numbers $R$, whose membership function $\mu_{A}$ satisfies the following conditions:

(i) $\mu_{A}$ is a mapping from $R$ to the closed interval $[0,1]$,

(ii) there exists a unique real number $m$ such that

(a) $\mu_{A}(m)=1$,

(b) $\mu_{A}$ is monotone nondecreasing on $(-\infty, m]$,

(c) $\mu_{A}$ is monotone nonincreesing on $[m,+\infty)$.

We call the number $m$ in (ii) the center of $A$, and denote the center of $A$ by $m_{\mathrm{A}}$, similarly the center of $B$ by $m_{B}$, etc. We should notice that no continuity of the membership function of the fuzzy number is assumed, but the uniqueness of the center is required unlike the definition of Dubois and Prade [3]. 
We denote the set of all fuzzy numbers defined as above by $\mathscr{F}$. Since the membership function of the real number, i.e. the characteristic function, satisfies the conditions of Definition 2.1, it holds that $R \subset \mathscr{F}$. The center of a real number $m$ is trivially $m$ itself.

An order relation among the fuzzy numbers is defined by the following.

Definition 2.2. For any fuzzy numbers $A, B \in \mathscr{F}, A \geqslant B$ iff

(i) $m_{B} \leq m_{A}$,

(ii) there exists a real number $c$ such that

(a) $m_{B} \leq c \leq m_{A}$,

(b) $\mu_{A}(x) \leq \mu_{B}(x) \quad \forall x<c$,

(c) $\mu_{A}(x) \geq \mu_{B}(x) \quad \forall x>c$.

$B \leqslant A$ is, of course, defined by $A \geqslant B$.

From the above definition we get the following elementary results.

Proposition 2.1. The order relation $\leqslant$ restricted on $R$ coincides with the usual order among the real numbers. That is, for any real numbers $\alpha$ and $\beta, \alpha \geqslant \beta$ iff $\alpha \geq \beta$.

Proof. It is trivial that if $\alpha \geqslant \beta$ then $\alpha \geq \beta$, since the centers of $\alpha$ and $\beta$ are equal to $\alpha$ and $\beta$, respectively. Conversly, if $\alpha \geq \beta$ then the condition (ii) of Definition 2.2 holds by taking any $c \in[\beta, \alpha]$.

Proposition 2.2. For any $\alpha \in R$ and any $A \in \mathscr{F}$,

(i) $\alpha \leqslant A$ iff $\mu_{A}(x)=0 \quad \forall x<\alpha$,

(ii) $A \leqslant \alpha$ iff $\mu_{A}(x)=0 \quad \forall x>\alpha$.

Proof. (i) Let $\alpha \leqslant A$, then $\alpha \leq m_{A}$ and there exists a real number $c \in\left[\alpha, m_{A}\right]$ such that

$$
\begin{aligned}
& \mu_{\mathrm{o}}(x) \geq \mu_{A}(x) \quad \forall x<c, \\
& \mu_{\mathrm{o}}(x) \leq \mu_{A}(x) \quad \forall x>c,
\end{aligned}
$$

Since $\alpha \leq c$, it follows from (2.1) that

$$
0=\mu_{\alpha}(x) \geq \mu_{A}(x) \quad \forall x<\alpha .
$$

Consequently $\mu_{A}(x)=0$ for $\forall x<\alpha$.

Conversely, let $\mu_{A}(x)=0$ for $\forall x<\alpha$. then $\alpha \leq m_{A}$ and

$$
\mu_{\alpha}(x)=\mu_{A}(x) \quad \forall x<\alpha .
$$

On the other hand it is trivial that

$$
0=\mu_{\alpha}(x) \leq \mu_{A}(x) \quad \forall x>\alpha .
$$

(2.3) and (2.4), together with the relation $\alpha \leq m_{A}$, imply $\alpha \leqslant A$.

The statement (ii) can be proved similarly.

Putting $\alpha=0$ in Proposition 2.2 we get the following.

Corollary 2.1. For any $A \in \mathscr{F}$ we have 


$$
\begin{aligned}
& \text { (i) } 0 \leqslant A \text { iff } \mu_{A}(x)=0 \quad \forall x<0, \\
& \text { (ii) } A \leqslant 0 \text { iff } \mu_{A}(x)=0 \quad \forall x>0 .
\end{aligned}
$$

REMARK 2.1. The right-hand statement in (2.5) coincides with the usual definition of a positive fuzzy number. Similarly the right-hand statement in (2.6) coincides with the usual definition of a negative fuzzy number. Therefore $0 \leqslant A$ (resp. $A \leqslant 0$ ) expresses that $A$ is a positive (resp. negative) fuzzy number referring to the usual definition.

Now we shall show a fundamental property of our order relation which is indispensable for all optimization problems related to the order relation.

THEOREM 2.1. The set F of fuzzy numbers is a partial ordered set with respect to the order relation introduced in Definition 2.2.

Proof. (Reflexivity) It is trivially true that $A \leqslant A$ for any $A \in \mathscr{F}$, since $\mu_{A}(x)=$ $\mu_{A}(x) \forall x \in R$.

(Asymmetricity) Let $A$ and $B$ be two fuzzy numbers satisfying both $A \leqslant B$ and $B$ $\leqslant A$. Then $m_{A} \leq m_{B} \leq m_{A}$, which implies $m_{A}=m_{B}$. Consequently, a number $c$ in (ii) of Definition 2.2 is uniquely determined as $c=m_{A}=m_{B}$ for either of $A \leqslant B$ and $B \leqslant A$.

Thus, from $\mathrm{A} \leqslant B$ we have

$$
\begin{aligned}
& \mu_{A}(x) \geq \mu_{B}(x) \quad \forall x<c, \\
& \mu_{A}(x) \leq \mu_{B}(x) \quad \forall x>c,
\end{aligned}
$$

and from $B \leqslant A$ we have

$$
\begin{aligned}
& \mu_{A}(x) \leq \mu_{B}(x) \quad \forall x<c, \\
& \mu_{A}(x) \geq \mu_{B}(x) \quad \forall x>c .
\end{aligned}
$$

From $(2.7) \sim(2.10)$ it follows that

$$
\mu_{A}(x)=\mu_{B}(x) \quad \forall x \neq c .
$$

Since $c=m_{A}=m_{B}$, it holds that $\mu_{A}(c)=\mu_{B}(c)=1$. Hence $\mu_{A}(x)=\mu_{B}(x) \forall x \in R$, which means $A=B$.

(Transitivity) Let $A, B$ and $C$ be fuzzy numbers, and let $A \leqslant B$ and $B \leqslant C$. Then $m_{A} \leq m_{B} \leq m_{C}$ and there exist a number $c_{1} \in\left[m_{A}, m_{B}\right]$ and a number $c_{2} \in\left[m_{B}, m_{C}\right]$ such that

$$
\begin{array}{ll}
\mu_{A}(x) \geq \mu_{B}(x) & \forall x<c_{1}, \\
\mu_{A}(x) \leq \mu_{B}(x) \quad \forall x>c_{1}, \\
\mu_{B}(x) \geq \mu_{C}(x) \quad \forall x<c_{2} \\
\mu_{B}(x) \leq \mu_{C}(x) \quad \forall x>c_{2} .
\end{array}
$$

Since $c_{1} \leq c_{2}$, it follows from $(2.11) \sim(2.14)$ that

$$
\mu_{A}(x) \geq \mu_{c}(x) \quad \forall x<c_{1},
$$




$$
\mu_{A}(x) \leq \mu_{c}(x) \quad \forall x>c_{2} .
$$

In the case that $c_{1}=c_{2}$, two relations (2.15) and (2.16) imply $A \leqslant C$.

We consider the case that $c_{1}<c_{2}$. From the definition of a fuzzy number, $\mu_{A}$ is nonincreasing on $\left[c_{1},+\infty\right)$ because $m_{A} \leq c_{1}$. Similarly, $\mu_{C}$ is nondecreasing on $\left(-x, c_{2}\right]$ because $c_{2} \leq m_{B}$. Hence $\mu_{A}$ is nonincreasing and $\mu_{C}$ is nondecreasing on the common interval $\left[c_{1}, c_{2}\right]$. From these monotonicities and (2.15), (2.16), we can choose a number $\bar{c} \in\left[c_{1}, c_{2}\right]$ satisfying

$$
\begin{array}{ll}
\mu_{A}(x) \geq \mu_{C}(x) & \text { on }\left[c_{1}, \bar{c}\right), \\
\mu_{A}(x) \leq \mu_{C}(x) & \text { on }\left(\bar{c}, c_{2}\right] .
\end{array}
$$

Combining four relations $(2.15),(2.16),(2.17)$ and (2.18) we get

$$
\begin{aligned}
& \mu_{A}(x) \geq \mu_{C}(x) \quad \forall x<\bar{c}, \\
& \mu_{A}(x) \leq \mu_{C}(x) \quad \forall x>\bar{c} .
\end{aligned}
$$

Thus the relation $A \leqslant C$ is proved.

At the end of this section, we like to emphasize that our order relation introduced here can be considered as a natural extension of the usual order among real numbers to the class of fuzzy numbers, owing to Proposition 2.1, Remark 2.1 and Theorem 2.1.

\section{Fundamental Operations on $L$ Fuzzy Numbers}

Definition 3.1. Let $L$ be a function from $R$ to $R$ satisfying the following conditions:

(i) $L(x)=L(-x) \quad \forall x \in R$,

(ii) $L(0)=1$,

(iii) $L(\cdot)$ is strictly decreasing on $[0,+\infty)$,

(iv) $\exists x_{0}>0$ such that $L\left(x_{0}\right)=0$.

Then the function $L$ is called a shape function, and the point $x_{0}$ in (iv) is called the zero point of $L$.

Definition 3.2. Let $m$ and $\alpha$ be any real numbers where $\alpha \neq 0$. And let $L$ be any shape function. $A$ fuzzy number $A$ whose membership function $\mu_{A}$ is expressed by the formula:

$$
\mu_{A}(x)=L\left(\frac{x-m}{\alpha}\right) \vee 0, \quad \mathbf{x} \in R
$$

is called an $L$ fuzzy number, where $a \vee b=\max (a, b)$.

By the definition in Section $2, m$ is equal to the center of $A$. We call $\alpha$ the deviation parameter of $A$.

Remark 3.1. According to the definition of Dubois and Prade [3], the deviation 
parameter of $L-R$ fuzzy number is restricted to have only the positive value. In contrast with their's, the deviation parameter is enlarged to the extent of negative values in our definition. This is for the purpose of introducing, later on, linear operations on the family of $L$ fuzzy numbers.

The graph of the membership function (3.1) concentrates around the vertical line $x$ $=m$ as $|\alpha|$ tends to zero. For $\alpha=0$, hence, we interpret the formula (3.1) as

$$
\mu_{A}(x)=\left\{\begin{array}{lll}
1 & \text { if } \quad x=m \\
0 & \text { if } \quad x \neq m
\end{array}\right.
$$

by considering the limit as tending $|\alpha|$ to zero. The formula (3.2) is no other than the characteristic function of the real number $m$. Then we define anew the $L$ fuzzy number as a fuzzy number whose membership function is given by either (3.1) or (3.2). For the sake of simplicity, we write the $L$ fuzzy number $A$ as

$$
A=(m, \alpha)_{L} \text {. }
$$

By the interpretation stated above, when $\alpha=0$ the $L$ fuzzy number $(m, 0)_{L}$ is equal to the real number $m$.

Given a shape function $L$, let us denote the set of all $L$ fuzzy numbers by

$$
\mathscr{F}_{L}=\left\{A=\left(m, \alpha_{L}\right) \mid m, \alpha \in R\right\} .
$$

Then we have $R \subset \mathscr{F}_{L}$ for every shape function $L$.

Now we shall introduce fundamental operations on $\mathscr{F}_{L}$ as follows.

Definition 3.3. Let $A=(m, \alpha)_{L}$ and $B=(n, \beta)_{L}$ be any $L$ fuzzy numbers. Then the sum $A \oplus B$ and the difference $A \ominus B$ are defined by (i) and (ii) respectively.

(i) $A \oplus B=(m+n, \alpha+\beta)_{L}$,

(ii) $A \ominus B=(m-n, \alpha-\beta)_{L}$.

The product of $A$ and a scalar $\lambda \in R$ is defined by

(iii) $\lambda \cdot A=(\lambda m, \lambda \alpha)_{L}$.

Next define an element $\theta$ of $\mathscr{F}_{L}$ by

$$
\theta=(0,0)_{L} .
$$

Then we have

Proposition 3.1. Let $L$ be an arbitrary shape function. Then $\mathscr{F}_{L}$ forms a linear space under the operations introduced in Definition 3.3, and the element $\theta$ given by (3.4) is the zero element of the linear space.

The proof of this proposition is very easy, and so is omitted.

Proposition 3.2. The fundamental operations restricted on $R$ coincide with the usual operations among real numbers. Namely we have

(i) $(m, 0)_{L} \oplus(n, 0)_{L}=(m+n, 0)_{L}$,

(ii) $(m, 0)_{L} \ominus(n, 0)_{L}=(m-n, 0)_{L}$, 
(iii) $\lambda \cdot(m, 0)_{L}=(\lambda m, 0)_{L}$.

The statements of this proposition are all direct from the definitions.

Our definitions of operations on $\mathscr{F}_{L}$ are considerably different from those of Dubois and Prade [3], which have been induced from a certain nonlinear binary relation. One will find later that the operations introduced in this paper are very convenient to develop subsequent various arguments.

Aside from the convenience of our operations, we have to give below some additional comments on the meanings of the operations.

Let $A=(m, \alpha)_{L}$ be any $L$ fuzzy number where $\alpha \neq 0$. Let $x_{0}$ be the zero point of $L$. Define a new variable $u$ by

$$
u=\frac{x-m}{\alpha}
$$

We consider first the case where $\alpha>0$. When we let the value of $x$ vary continuously from $m-\alpha x_{0}$ to $m+\alpha x_{0}$, the point $(u, L(u))$ of $R^{2}$ moves from $\left(-x_{0}, 0\right)$ to $\left(x_{0}, 0\right)$ in the clockwise direction along the curve of the shape function $L$. Then we say that the fuzzy number $A$ has the clockwise direction. In the case where $\alpha<0$, if we let the value of $\mathrm{x}$ vary continuously from $m-|\alpha| x_{0}$ to $m+|\alpha| x_{0}$, then the point $(u, L(w))$ moves from $\left(x_{0}, 0\right)$ to $\left(-x_{0}, 0\right)$ in the counterclockwide direction along the curve $L$. Hence, in this case, we say that $A$ has the counterclockwise direction.

Now, the graph of the membership function $\mu_{A}$ restricted on its support is expressed by the set

$$
\left\{\left(x, L\left(\frac{x-m}{\alpha}\right)\right)|m-| \alpha\left|x_{0}<x<m+\right| \alpha \mid x_{0}\right\} .
$$

We shall identify all sets obtained by translating the set (3.5) in the direction of $x$-axis. By $\mathscr{G}_{A}$ we shall denote the equivalent class obtained by translation of the set (3.5) in the direction of $x$-axis.

Let $A=(m, \alpha)_{L}$ be an arbitrary $L$ fuzzy number. By our operation, $-A=(-m$, $-\alpha)_{L}$. By virtue of the symmetricity of $L$, the graph of the membership function of $-A$ restricted on its support is related to the graph of $\mu_{A}$ as follows:

$$
\begin{aligned}
& \left\{\left(x, L\left(\frac{x+m}{-\alpha}\right)\right)|-m-| \alpha\left|x_{0}<x<-m+\right| \alpha \mid x_{0}\right\} \\
= & \left\{\left(x, L\left(\frac{x-m}{\alpha}\right)\right)|m-| \alpha\left|x_{0}<x<m+\right| \alpha \mid x_{0}\right\}-(2 m, 0) .
\end{aligned}
$$

Since, by our definition $\mathscr{G}_{A}$ is invariant under the translation in the $x$-axis direction, (3.6) implies that

$$
\mathscr{G}_{(-A)}=\mathscr{G}_{A}
$$

We shall denote anew the equivalent class generated by the fuzzy number $A$ by $\mathscr{G}_{|A|}$. then we have from (3.7) that

$$
\mathscr{G}_{|A|}=\mathscr{G}_{A}=\mathscr{G}_{(-A)}
$$


When we like to show explicitly the direction of the fuzzy number in the formula (3.3), we write

$$
A=\left(m, \widehat{\mathscr{G}_{|A|}}\right)_{L}
$$

if $A$ has the clockwise direction, and

$$
A=\left(m, \check{G}_{|A|}\right)_{L}
$$

if $A$ has the counterclockwise direction.

Proposition 3.3. Let $A=(m, \alpha)_{L}$. Then it holds that

$$
\begin{aligned}
& \text { (i) if } A=\left(m, \widetilde{\mathscr{G}_{|A|}}\right)_{L}, \text { then }-\mathrm{A}=\left(-m, \widetilde{\mathscr{G}_{|A|}}\right)_{L}, \\
& \text { (ii) if } A=\left(m, \widetilde{\mathscr{G}_{|A|}}\right)_{L}, \text { then }-\mathrm{A}=\left(-m, \widetilde{\mathscr{G}_{|A|}}\right)_{L} .
\end{aligned}
$$

Proof. Easy from our definitions.

Now consider an arbitrary $L$ fuzzy number $A=(m, \alpha)_{L}$. Since $\mathscr{F}_{L}$ forms a linear space as stated in Proposition 3.1, it holds that

$$
A \oplus(\ominus A)=A \oplus(-A)=A \ominus A=\theta .
$$

One may perhaps feel strange in the formula (3.11) which shows that, although the graphs of $\mu_{A}$ and $\mu_{(-A)}$ are completely same except their locations, the sum of $A$ and $-A$ is equal to the zero element of $\mathscr{F}_{L}$ neverthless. This fact may be considered as reasonable, if one find in Proposition 3.3 that $A$ and $-A$ are in the oposite sides of the direction and so their directed graphs are cancelled out each other. That is, symbolically writing, when $A$ has the clockwise direction we have from Proposition 3.3 (i) the following result of an addition:

$$
\begin{aligned}
A & =\left(m, \widehat{\mathscr{G}_{|A|}}\right)_{L} \\
\oplus \quad-A & =\left(-m, \overleftrightarrow{\mathscr{G}_{|A|}}\right)_{L} \\
\hline A \ominus A & =(0,0)_{L}
\end{aligned}
$$

We get a similar result too in the case where $A$ has the counterclockwise direction.

\section{An Order Relation of $L$ Fuzzy Numbers}

In this section we shall apply the order relation defined in Section 2 to the family $\mathscr{F}_{L}$ of $L$ fuzzy numbers, and give a characterization of the order in terms of the parameters of $L$ fuzzy numbers. Let $L$ be an arbitrary shape function, and let $x_{0}$ the zero point of $L$.

TheOREM 4.1. Let $A=(m, \alpha)_{L}$ and $B=(n, \beta)_{L}$ be $L$ fuzzy numbers such that $\alpha \beta \geq 0$. Then we have

$$
A \geqslant B \Leftrightarrow\left\{\begin{array}{l}
m \geq n, \\
|\alpha-\beta| \leq(m-n) / x_{0} .
\end{array}\right.
$$

Proof. It is direct from Definition 2.2 that if $A \geqslant B$, then $m \geq n$. Therefore, in 
order to prove (4.1), it suffices to show the relation:

$$
A \geqslant B \Leftrightarrow|\alpha-\beta| \leq(m-n) / x_{0}
$$

under the assumption that $m \geq n$.

Step 1: Find a necessary and sufficient condition for the inequality

$$
L\left(\frac{x-m}{\alpha}\right) \leq L\left(\frac{x-n}{\beta}\right)
$$

to hold everywhere on $(-\infty, n]$.

Case 1: $\quad \alpha>\beta>0$.

Two variables $(x-m) / \alpha$ and $(x-\mathrm{n}) / \beta$ are both nonpositive for $\forall x \leq n$, and $L$ is strictly increasing on $(-\infty, 0]$. Therefore, the inequality $(4.3)$ restricted on $(-\infty, n]$ is equivalent to

$$
(x-m) / \alpha \leq(x-n) / \beta .
$$

Let

$$
t_{0}=(\alpha n-\beta m) /(\alpha-\beta) .
$$

Then we have $t_{0} \leq n$ and the range of $x$ satisfying (4.4) on $(-x, n]$ is given by the interval $\left[t_{0}, n\right]$.

On the other hand, the unique solution of the equation

$$
L\left(\frac{x-m}{\alpha}\right)=0 \quad \text { on }(-\infty, m]
$$

is given by $x_{T}=m-\alpha x_{0}$. In order that (4.3) holds everywhere on $(-\infty, n]$, it is necessary and sufficient that the relation

$$
t_{0} \leq x_{T}
$$

holds. Finally, from (4.7) we get the relation

$$
\alpha-\beta \leq(m-n) / x_{0},
$$

as a necessary and sufficient condition to be required.

Case 2: $\beta>\alpha>0$.

Solving (4.3) on the interval $(-\infty, n]$, we get $x \leq t_{0}$, where $t_{0}$ is defined by (4.5). Since the relation $t_{0} \geq n$ holds in this case, we see that the inequality (4.3) holds everywhere on $(-x, n]$ without any other condition to be added.

Case 3: $\alpha=\beta>0$.

It holds that

$$
(x-m) / \alpha \leq(x-n) / \alpha \leq 0 \quad \forall x \leq n .
$$

Since $L$ is strictly increasing on $(-\infty, 0]$, it follows from (4.9) that the inequality (4.3) holds for all $x \leq n$.

Step 2: Find a necessary and sufficient condition for the inequality 


$$
L\left(\frac{x-m}{\alpha}\right) \geq L\left(\frac{x-n}{\beta}\right)
$$

to holds everywhere on $[\mathbf{m}+x)$.

Case 1: $\alpha>\beta>0$.

Two functions $(x-m) / \alpha$ and $(x-n) / \beta$ are both nonnegative for $\forall x \geq m$, and $L$ is strictly decreasing on $[0,+\infty)$. Hence, by solving the inequality $(4.10)$ on $[m,+\infty)$, we have that $x \geq t_{0}$. Since $t_{0} \leq m$ in the case, we see that the inequality (4.10) holds everywhere on $[m,+\infty)$ without any other condition.

Case 2: $\beta>\alpha>0$.

In this case, we have $t_{0} \geq m$ and the range of $x$ satisfying $(4.10)$ on $[m,+\infty)$ is given by $\left[m, t_{0}\right]$. The unique solution of the equation

$$
L\left(\frac{x-n}{\beta}\right)=0 \text { on }[n,+\infty)
$$

is $x_{s}=n+\beta x_{0}$. In order that (4.10) holds everywhere on $[m,+\infty)$, it is necessary and sufficient that the relation

$$
x_{s} \leq t_{0}
$$

holds. Hence, from (4.12) we get the relation

$$
\alpha-\beta \geq-(m-n) / x_{0}
$$

as a required condition.

Case 3: $\alpha=\beta>0$.

It holds that

$$
0 \leq(x-m) / \alpha \leq(x-n) / \alpha \quad \forall x \geq m .
$$

Since $L$ is strictly decreasing on $[0,+\infty)$, we get from (4.14) that the inequality $(4.10)$ holds for all $x \geq m$.

Summing up all results above, we get the relation (4.1) when $\alpha$ and $\beta$ are both positive. Especially when $\alpha=\beta>0$ it holds that

$$
A \geqslant B \Leftrightarrow m \geq n,
$$

which follows from Case 3 .

Now, if $\alpha=0$ then the membership function of $A$ is the characteristic function $c_{m}(\cdot)$ of the real number $m$.

Case 4: $\alpha=0$ and $\beta \neq 0$.

It is trivially true that

$$
0=c_{m}(x) \leq L\left(\frac{x-n}{\beta}\right) \quad \forall x \leq n,
$$

because $m \geq n$. Hence we have only to find a necessary and sufficient condition for the inequality

$$
\left\llcorner\left(\frac{x-n}{\beta}\right) \leq c_{m}(x) \quad \forall x \geq m\right.
$$


to hold.

In the case where $\beta>0$, the unique solution of (4.11) is $x_{s}=n+\beta x_{0}$. Since (4.15) is equivalent to that $x_{s} \leq m$, we obtain the condition

$$
\beta \leq(m-n) / x_{0} .
$$

Similary, in the case where $\beta<0$, we get the condition

$$
-\beta \leq(m-n) / x_{0} \text {. }
$$

Combining (4.16) with (4.17) we have that

$$
A \geqslant B \Leftrightarrow\left\{\begin{array}{l}
m \geq n, \\
|\beta| \leq(m-n) / x_{0} .
\end{array}\right.
$$

This relation is surely equal to (4.1) in which $\alpha=0$ is substituted.

Case 5: $\alpha \neq 0$ and $\beta=0$.

In the same way as Case 4 we get

$$
A \geqslant B \Leftrightarrow\left\{\begin{array}{l}
m \geq n \\
|\alpha| \leq(m-n) / x_{0}
\end{array}\right.
$$

Finally, in the case where $\alpha<0$ and $\beta<0$, we can show the relation (4.1) in the same way as the case where $\alpha>0$ and $\beta>0$. Thus we have completed the proof.

In order to derive a relation similar to (4.1) in the case where $\alpha \beta<0$, we need to prepare the following.

Proposition 4.1. Let $m, n, \alpha$ and $\beta$ be arbitrary real numbers. Then it holds that

$$
\begin{aligned}
(m, \alpha)_{L} \geqslant(n, \beta)_{L} & \Leftrightarrow(m, \alpha)_{L} \geqslant(n,-\beta)_{L} \\
& \Leftrightarrow(m,-\alpha)_{L} \geqslant(n, \beta)_{L} \\
& \Leftrightarrow(m,-\alpha)_{L} \geqslant(n,-\beta)_{L} .
\end{aligned}
$$

Proof. By the definition of the order,

$$
\begin{aligned}
(m, \alpha)_{L} \geqslant & (n, \beta)_{L} \\
& \Leftrightarrow\left\{\begin{array}{l}
m \geq n \text { and } \\
n \leq \exists c \leq m \text { such that } \\
L\left(\frac{x-m}{\alpha}\right) \leq L\left(\frac{x-n}{\beta}\right) \quad \forall x<c, \\
L\left(\frac{x-m}{\alpha}\right) \geq L\left(\frac{x-n}{\beta}\right) \quad \forall x>c .
\end{array}\right.
\end{aligned}
$$

By the symmetricity of $L,(4.18)$ and (4.19) are equivalent to

$$
L\left(\frac{x-m}{\alpha}\right) \leq L\left(\frac{x-n}{-\beta}\right) \quad \forall x<c
$$

and 


$$
L\left(\frac{x-m}{\alpha}\right) \geq L\left(\frac{x-n}{-\beta}\right) \quad \forall x>c
$$

respectively. From these relations we have

$$
(m, \alpha)_{L} \geqslant(m, \beta)_{L} \Leftrightarrow(m, \alpha)_{L} \geqslant(n,-\beta)_{L} .
$$

The other statements can be proved similary.

THEOREM 4.2. Let $A=(m, \alpha)_{L}$ and $B=(n, \beta)_{L}$ be $L$ fuzzy numbers such that $\alpha \beta<0$. Then we have

$$
A \geqslant B \Leftrightarrow\left\{\begin{array}{l}
m \geq n \\
|\alpha+\beta| \leq(m-n) / x_{0}
\end{array}\right.
$$

Proof. By Proposition 4.1 we have

$$
(m, \alpha)_{L} \geqslant(n, \beta)_{L} \Leftrightarrow(m, \alpha)_{L} \geqslant(n,-\beta)_{L} .
$$

By the assumption we have $\alpha(-\beta)>0$. Applying Theorem 4.1 to the right-hand side of (4.21), hence, we get

$$
(m, \alpha)_{L} \geqslant(n,-\beta)_{L} \Leftrightarrow\left\{\begin{array}{l}
m \geq n, \\
|\alpha+\beta| \leq(m-m) / x_{0} .
\end{array}\right.
$$

Combining (4.21) with (4.22) implies (4.20), which completes the proof.

In the rest of this section, we shall give several rules of fundamental operations among $L$ fuzzy numbers in the case where the order relation is participating.

Proposition 4.2. Let $A=(m, \alpha)_{L}$ be an $L$ fuzzy number, and let $\theta$ be the zero element of $\mathscr{F}_{L}$ defined by (3.4). Then

$$
\begin{aligned}
& \text { (i) } A \geqslant \theta \Leftrightarrow\left\{\begin{array}{l}
m \geq 0 \\
|\alpha| \leq m / x_{0} .
\end{array}\right. \\
& \text { (ii) } A \leqslant \theta \Leftrightarrow\left\{\begin{array}{l}
m \leq 0 \\
|\alpha| \leq(-m) / x_{0} .
\end{array}\right. \\
& \text { (iii) } A \geqslant \theta \Leftrightarrow-A \leqslant \theta . \\
& \text { (iv) } A \geqslant \theta \Rightarrow \forall \lambda \geq 0, \quad \lambda A \geqslant \theta . \\
& \text { (v) } A \geqslant \theta \Rightarrow \forall \lambda<0, \quad \lambda A \leqslant \theta .
\end{aligned}
$$

Proof. (i) is direct from putting $B=\theta$ in Theorem 4.1, and (ii) is derived from putting $A=\theta$ first and then putting $A$ in place of $B$ in the same theorem.

(iii) Substitute $-A$ for $A$ in (ii), then

$$
-A \leqslant \theta \Leftrightarrow\left\{\begin{array}{l}
-m \leq 0 \\
|\alpha| \leq m / x_{0}
\end{array}\right.
$$

The statement of (ii) is obvious from (i) and (4.23).

(iv) From the right-hand side of (i), for any $\lambda \geq 0$ it holds that 


$$
\lambda m \geq 0 \text { and } \lambda|\alpha|=|\lambda \alpha| \leq \lambda m / x_{0},
$$

which implies $(\lambda m, \lambda \alpha)_{L} \geqslant \theta$ by (i) again. Namely $\lambda A \geqslant \theta$.

(v) Let $A \geqslant \theta$. By (ii), then, for any $\lambda<0$ it holds that

$$
-\lambda m \geq 0 \text { and } \lambda|\alpha| \geq \lambda m / x_{0},
$$

hence

$$
\lambda m \leq 0 \text { and }|\lambda \alpha| \leq-\lambda m / x_{0} .
$$

This implies $(\lambda m, \lambda \alpha)_{L} \leqslant \theta$. Namely $\lambda A \leqslant \theta$.

Proposition 4.3 For two L fuzzy numbers $A=(m, \alpha)_{L}$ and $B=(n, \beta)_{L}$ such that $A \geqslant B$, it holds that

$$
\begin{aligned}
& \text { (i) } \quad \lambda A \geqslant \lambda B, \quad \forall \lambda>0, \\
& \text { (ii) } \mu A \leqslant \mu B, \quad \forall \mu<0 .
\end{aligned}
$$

Proof. We prove only (ii), since (i) is proved similarly.

Let $\alpha \beta \geq 0$. By the assumption we have

$$
m \geq n \text { and }|\alpha-\beta| \leq(m-n) / x_{0} .
$$

For $\mu<0$, then, it holds that

$$
\mu m \leq \mu n \text { and }|\mu \alpha-\mu \beta|=-\mu|\alpha-\beta| \geq(\mu n-\mu m) / x_{0} .
$$

Since

$$
(\mu \alpha)(\mu \beta)=\mu^{2} \alpha \beta \geq 0,
$$

(4.24) implies that $\mu A \leqslant \mu B$ by virtue of Theorem 4.1 .

In the case where $\alpha \beta<0$, we have

$$
m \geq n \text { and }|\alpha+\beta| \leq(m-n) / x_{0} .
$$

For $\mu<0$, then, we have

$$
\mu m \leq \mu n \text { and }|\mu \alpha+\mu \beta|=-\mu|\alpha+\beta| \geq(\mu n-\mu \mathrm{m}) / x_{0} .
$$

Since $(\mu \alpha)(\mu \beta)=\mu^{2} \alpha \beta<0$, (4.25) implies $\mu A \leqslant \mu B$ by Theorem 4.2. This completes the proof.

Define $\mathscr{K}_{L}$ by the following:

$$
\mathscr{K}_{L}=\left\{A \in \mathscr{F}_{L} \mid A \geqslant \theta\right\} .
$$

Proposition 4.4. $\mathscr{K}_{L}$ is a convex cone in the linear space $\mathscr{F}_{L}$. Namely it holds that

(i) $A \in \mathscr{K}_{L} \Rightarrow \lambda A \in \mathscr{K}_{L} \quad \forall \lambda \geq 0$,

(ii) $A, B \in \mathscr{K}_{L} \Rightarrow A \oplus B \in \mathscr{K}_{L}$.

Proof. (i) is no other than Proposition 4.2(iv).

(ii) Let $A=(m, \alpha)_{L} \in \mathscr{K}_{L}$ and let $B=(n, \beta)_{L} \in \mathscr{K}_{L}$. By Proposition 4.2(i), then 
we have

$$
m \geq 0,|\alpha| \leq m / x_{0}
$$

and

$$
n \geq 0,|\beta| \leq n / x_{0},
$$

which yield that

$$
\left\{\begin{array}{l}
m+n \geq 0, \\
|\alpha+\beta| \leq|\alpha|+|\beta| \leq(m+n) / x_{0} .
\end{array}\right.
$$

Since $A \oplus B=(m+n, \alpha+\beta)_{L},(4.27)$ implies by Proposition 4.2 (i) again that $A \oplus B \in \mathscr{K}_{L}$. This completes the proof.

Proposition 4.5. Let $A=(m, \alpha)_{L}$ and $B=(n, \beta)_{L}$. Suppose that $\alpha \beta \geq 0$. Then

$$
A \geqslant B \Leftrightarrow A \ominus B \geqslant \theta \Leftrightarrow B \ominus A \leqslant \theta .
$$

Proof. By virtue of Theorem 4.1 it holds that

$$
A \geqslant B \Leftrightarrow\left\{\begin{array}{l}
m \geq n, \\
|\alpha-\beta| \leq(m-n) / x_{0} .
\end{array}\right.
$$

Recalling that $A \ominus B=(m-n, \alpha-\beta)_{L}$, by Proposition 4.2(i) we have

$$
A \ominus B \geqslant \theta \Leftrightarrow\left\{\begin{array}{l}
m-\mathrm{n} \geq 0 \\
|\alpha-\beta| \leq(m-n) / x_{0} .
\end{array}\right.
$$

The left half of (4.28) is obvious from (4.29) and (4.30).

Next, from Proposition 4.2 (iii) it follows that

$$
A \ominus B \geqslant \theta \Leftrightarrow-A \ominus B \leqslant \theta .
$$

Since $-A \ominus B=B \ominus A$, (4.31) is equivalent ot the relation

$$
A \ominus B \geqslant \theta \Leftrightarrow B \ominus A \leqslant \theta,
$$

which is the right half of (4.28).

Proposition 4.6. Let $A=(m, \alpha)_{L}$ and $B=(m, \beta)_{L}$. Suppose that $\alpha \beta<0$. Then

$$
A \ominus B \geqslant \theta \Rightarrow A \geqslant B \text {. }
$$

Proof. By Proposition 4.2 (i) we have

$$
A \ominus B \geqslant \theta \Leftrightarrow\left\{\begin{array}{l}
m-n \geq 0 \\
|\alpha-\beta| \leq(m-n) / x_{0} .
\end{array}\right.
$$

In the case where $\beta<0<\alpha$, we have

$$
|\alpha+\beta| \leq|\alpha|+|\beta|=\alpha-\beta=|\alpha-\beta| .
$$

Frome (4.33) and (4.34) it follows that 


$$
A \ominus B \geqslant \theta \Rightarrow\left\{\begin{array}{l}
m-n \geq 0 \\
|\alpha+\beta| \leq(m-n) / x_{0} .
\end{array}\right.
$$

The right hand side of (4.35) is equivalent to the relation that $A \geqslant B$ by virtue of Theorem 4.2. This proves (4.32) in this case.

The case where $\alpha<0<\beta$ is proved similarly.

The following three propositions can be proved easily by using Theorem 4.1 or Theorem 4.2, ans so their proofs are omitted.

Proposition 4.7. Let $A=(m, \alpha)_{L}, B=(n, \beta)_{L}$ and $C=(\ell, \gamma)_{L}$, where $\alpha>0$, $\beta>0$ and $\gamma>0$. Then we have the following:

(i) If $\beta \geq \gamma$, then

$$
A \oplus C \geqslant B \Leftrightarrow A \geqslant B \ominus C .
$$

(ii) If $\alpha \geq \gamma$, then

$$
A \geqslant B \oplus C \Leftrightarrow A \ominus C \geqslant B
$$

Proposition 4.8. Let $A=(m, \alpha)_{L}, B=(n, \beta)_{L}$ and $C=(\ell, \gamma)_{L}$, where $\alpha>0$, $\beta>0$ and $\gamma>0$. Then we have the following:

(i) If $\gamma \leq \min \{\alpha, \beta\}$ or $\max \{\alpha, \beta\} \leq \gamma$, then

$$
A \geqslant B \Leftrightarrow A \ominus C \geqslant B \ominus C .
$$

(ii) If $\beta<\gamma<\alpha$ or $\alpha<\gamma<\beta$, then

$$
A \geqslant B \Rightarrow A \ominus C \geqslant B \ominus C \text {. }
$$

Proposition 4.9. Let $A=(m, \alpha)_{L}, B=(n, \beta)_{L}$ and $C=(\ell, \gamma)_{L}$, where $\gamma>0$. If any one of the following conditions $(\mathrm{i}) \sim(\mathrm{v})$ is satisfied, it holds that

$$
A \geqslant B \Rightarrow A \oplus C \geqslant B \oplus C \text {. }
$$

(i) $\alpha>0$ and $\beta>0$.

(ii) $\beta<0<\alpha$ and $\beta+\gamma<0$.

(iii) $\alpha<0<\beta$ and $\alpha+\gamma<0$.

(iv) $\alpha<0, \beta<0, \alpha+\gamma<0$ and $\beta+\gamma<0$.

(v) $\alpha<0, \beta<0, \alpha+\gamma>0$ and $\beta+\gamma>0$.

\section{Applications to Fuzzy Linear Programming}

For every shape function $L$, we define the subset $\mathscr{F}_{L}^{+}$by

$$
\mathscr{F}_{L}^{+}=\left\{A=(m, \alpha)_{L} \mid m \in R, \alpha \geq 0\right\} .
$$

Namely, each member of $\mathscr{F}_{L}^{+}$is an $L$ fuzzy number having a nonnegative deviation parameter. 
We shall introduce several real constants and fuzzy numbers needed for the fuzzy linear programming model to be described.

Let $c_{1}, c_{2}, \ldots, c_{n}$ be real constants. Let $L_{1}, L_{2}, \ldots, L_{m}$ be $m$ shape functions, and for each $i(1 \leq i \leq m)$ let $x_{i 0}$ denote the zero point of the shape function $L_{i}$. For each $i(1 \leq i \leq m)$, let $A_{i 1}, A_{i 2}, \ldots, A_{i n}$ be $n L_{i}$-fuzzy numbers such that

$$
A_{i j}=\left(m_{i j}, \alpha_{i j}\right)_{L_{i}} \in \mathscr{F}_{L_{i}}^{+}, j=1,2, \ldots, n .
$$

In addition, another set of fuzzy numbers are given:

$$
B_{i}=\left(n_{i}, \beta_{i}\right)_{L_{i}} \in \mathscr{F}_{L_{i}}^{+}, \quad i=1,2, \ldots, m .
$$

In what follows, real constants $\left\{c_{i}\right\}$ and shape functions $\left\{L_{i}\right\}$ are arbitrarily given, and fuzzy numbers $\left\{A_{i j}\right\},\left\{B_{i}\right\}$ are arbitrary so far as their deviation parameters satisfy the nonnegativity.

Now we consider the first problem of the fuzzy linear programming given by the following:

$$
(F L P 1) \quad\left\{\begin{array}{l}
\text { minimize } \sum_{i=1}^{n} c_{i} x_{i} \\
\text { subject to } \\
x_{1} A_{i 1} \oplus x_{2} A_{i 2} \oplus \cdots \oplus x_{n} A_{\text {in }} \leqslant B_{i}, \\
i=1,2, \ldots, m \\
x_{j} \geq 0, \quad j=1,2, \ldots, n .
\end{array}\right.
$$

By the definition of the operations, we have

$$
\begin{aligned}
x_{1} A_{i 1} & \oplus x_{2} A_{i 2} \oplus \ldots \oplus x_{n} A_{i n} \\
& =\sum_{j=1}^{n} x_{j}\left(m_{i j}, \alpha_{i j}\right)_{L_{i}} \\
& =\left(\sum_{j=1}^{n} m_{i j} x_{j}, \sum_{j=1}^{n} \alpha_{i j} x_{j}\right)_{L_{i}} .
\end{aligned}
$$

For $\left\{x_{j}\right\}$ satisfying (5.5), it holds that

$$
\sum_{j=1}^{n} \alpha_{i j} x_{j} \geq 0, \quad i=1,2, \ldots, m,
$$

by virtue of (5.1). We have also that $\beta_{i} \geq 0 \forall i$ by (5.2). Then the constraint (5.4) is transformed by Theorem 4.1 as follows.

$$
\begin{aligned}
(5.4) & \Leftrightarrow\left(\sum_{j=1}^{n} m_{i j} x_{j}, \sum_{j=1}^{n} \alpha_{i j} x_{j}\right)_{L_{i}} \leqslant\left(n_{i}, \beta_{i}\right)_{L_{i}} \\
& \Leftrightarrow\left\{\begin{array}{l}
n_{\mathrm{i}}-\sum_{j=1}^{n} m_{i j} x_{j} \geq 0, \\
\left|\sum_{j=1}^{n} \alpha_{i j} x_{j}-\beta_{i}\right| \leq\left(n_{i}-\sum_{j=1}^{n} m_{i j} x_{j}\right) / x_{i 0} .
\end{array}\right.
\end{aligned}
$$

Rewriting (5.7), we obtain the following theorem.

THEOREM 5.1. The problem (FLP1) is equivalent to the problem: 


$$
(L P 1)\left\{\begin{array}{l}
\text { minimize } \sum_{i=1}^{n} c_{j} x_{j} \\
\text { subject to } \\
\sum_{j=1}^{n} m_{i j} x_{j} \leq n_{i}, i=1,2, \ldots, m \\
\sum_{j=1}^{n}\left(m_{i j}+x_{i 0} \alpha_{i j}\right) x_{j} \leq n_{i}+x_{i 0} \beta_{i}, \\
\sum_{j=1}^{n}\left(m_{i j}-x_{i 0} \alpha_{i j}\right) x_{j} \leq n_{i}-x_{i 0} \beta_{i}, \\
\quad i=1,2, \ldots, m \\
x_{j} \geq 0, j=1,2, \ldots, n .
\end{array}\right.
$$

The problem $(L P 1)$ is no longer a fuzzy problem, but is a linear programming problem in the usual sense. Therefore, the above theorem shows us that the fuzzy problem $(F L P 1)$ can be solved by applying the usual method of the linear programming to $(L P 1)$.

Even if, in the constraint (5.4), we adopt the order relation introduced by Dubois and Prade [5] or Prade [7] in place of our order relation, the fuzzy problem (FLP1) can be transformed to a nonfuzzy linear programming problem as shown in $[2,6,8]$. However, the nonfuzzy linear program transformed by adopting their concept does depend on the choice of the level for the fuzzy coefficients in the constraint of the original problem. On the other hand, the problem $(L P 1)$ is obviously not concerned with any level value. Namely, in our case, the transformation from the problem to the nonfuzzy one can be accomplished without help of the level value.

The second problem we treat is the case where the coefficients of the objective function in (FLP1) are also fuzzy numbers. Let $L_{0}$ be an arbitrary shape function having the zero point $x_{0}$, and let $C_{1}, \ldots, C_{n}$ be $n L_{0}$-fuzzy numbers such that

$$
C_{i}=\left(l_{i}, \gamma_{i}\right)_{L_{0}} \in \mathscr{F}_{L_{i}}^{+}, \quad i=1,2, \ldots, n .
$$

Let $\left\{A_{i j}\right\}$ and $\left\{B_{i}\right\}$ be the same as in the problem (FLP1).

Define the second problem of the fuzzy linear programming as follows:

$$
(F L P 2)\left\{\begin{array}{l}
\text { minimize } \sum_{i=1}^{n} x_{i} C_{i} \\
\text { subject to } \\
x_{1} A_{i 1} \oplus x_{2} A_{i 2} \oplus \ldots \oplus x_{n} A_{i n} \leqslant B_{i}, \\
i=1,2, \ldots, m \\
x_{j} \geq 0, j=1,2, \ldots, n .
\end{array}\right.
$$

In the above problem, the minimizing (5.9) is interpreted to minimize with respect to the order relation defined in Section 2. Of course, the smallest solution does not necessarily exist, because the order relation is a partial order.

We define the set of feasible solutions of (FLP2) by 


$$
S=\left\{\begin{array}{l|l}
x=\left(x_{1}, x_{2}, \ldots, x_{n}\right)^{T} \mid \begin{array}{c}
x_{1} A_{i 1} \oplus x_{2} A_{i 2} \oplus \ldots \oplus x_{n} A_{i n} \leqslant B_{i} \\
i=1,2, \ldots, m \\
x_{j} \geq 0, j=1,2, \ldots, n
\end{array}
\end{array}\right\} .
$$

As shown in the case of $(F L P 1), x=\left(x_{1}, x_{2}, \ldots, x_{n}\right)^{T}$ belongs to the set $S$ iff the following inequalities hold:

$$
\begin{aligned}
& \sum_{j=1}^{n}\left(m_{i j}+x_{i 0} \alpha_{i j}\right) x_{j} \leq n_{i}+x_{i 0} \beta_{i}, i=1,2, \ldots, m . \\
& \sum_{\mathrm{j}=1}^{n}\left(m_{i j}-x_{i 0} \alpha_{i j}\right) x_{j} \leq n_{i}-x_{i 0} \beta_{i}, \\
& x_{j} \geq 0, j=1,2, \ldots, n .
\end{aligned}
$$

Hence it is trivial that $S$ is a convex polyhedron in $R^{n}$.

Let

$$
C=\left(C_{1}, C_{2}, \ldots, C_{n}\right)^{T}
$$

where $\left\{C_{i}\right\}$ is given in (5.8).

Definition 5.1. A feasible solution $x^{*} \in S$ is said to be an efficient solution of $(F L P 2)$ iff $x^{*}$ satisfies the following relation:

$$
x \in S, C^{T} x \leqslant C^{T} x^{*} \Rightarrow C^{T} x=C^{T} x^{*} .
$$

Finally we get:

THEOREM 5.2. To find an efficient solution tó (FLP2) is equivalent to solve the following problem:

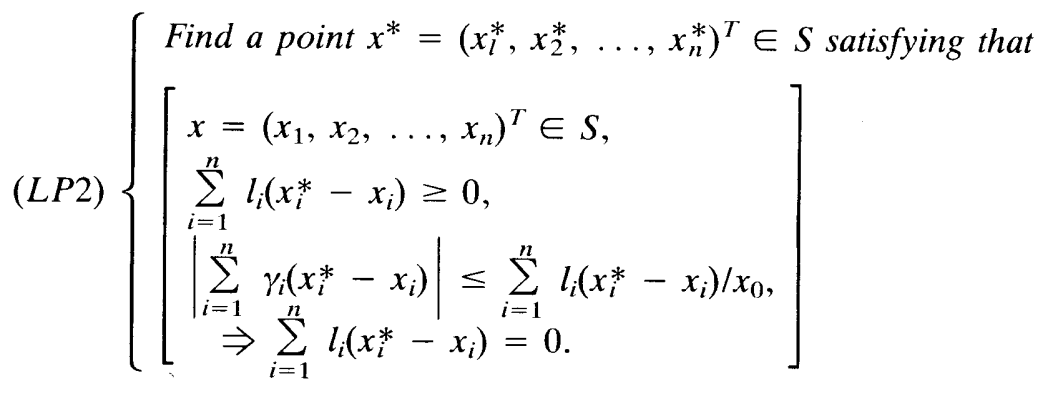

Proof. By Theorem 4.1 it is easily shown that (5.10) is equivalent to (5.11).

Owing to Theorem 5.2, the fuzzy problem (FLP2) as well as (FLP1) can be reduced to a nonfuzzy problem, which is described by only a set of linear inegualities. However, in contrast with the case of $(F L P 1)$, the reduced problem ( $L P 2)$ is not a linear programming problem in the usual sense. The problem seems to have a similarity with the multiobjective linear program. How to solve the problem (LP2) remains open in the present paper. 


\section{Concluding Remarks}

In this paper we have restricted the fuzzy number to the one which is symmetrical about its center, that is, the $L$ fuzzy number given by Definition 3.2 . That is only for the sake of the symplicity of arguments. Similar results can be obtained by following the manner of this paper, even when we admit unsymmetrical ones into the class of fuzzy numbers.

Among the various kinds of results obtained from Section 2 through Section 4, only some of them have been used for the fuzzy linear programming problems presented in the last section. For instance, Theorem 4.2 has not been needed for the application to the problems. Many of the results, including Theorem 4.2, which have not been utilized in Section 5 are to be substantially utilized to develop a theory of nonlinear fuzzy programming in the author's paper in preparation.

\section{References}

[1] Bellman R. and Zadeh L. A.: Decision-making in a fuzzy environment. Management Science, 17 (1970), 141-164.

[2] Dubors D.: Linear programming with fuzzy data. In "Analysis of Fuzzy Information" (J. C. Bezdek, ed.), 3, 241-263, CRC Press, Boca Raton, FL, 1987.

[3] Dubois D. and Prade H.: Operations on fuzzy numbers. Int. J. Systems Science, 9 (1978), 613-626.

[4] Dubors D. and Prade H.: Systems of linear fuzzy constraints., Fuzzy sets and Systems, 3 (1980), $37-48$.

[5] Dubois D. and Prade H.: Ranking fuzzy numbers in the setting of possibility theory. Information Sciences, 30 (1983), 183-224.

[6] Inuiguchi M. and Ichinashi H.: Relative modalities and their use in possibilistic linear programming. Fuzzy Sets and Systems, 35 (1990), 303-323.

[7] PrAde H.: Modal semantics and fuzzy set theory. In "Fuzzy Set and Possibility Theory" (R. R. Yager, ed.), 232-246, Pergamon Press, Oxford-New York, 1982.

[8] TANAKa H. and Asal K.: Fuzzy linear programming problems with fuzzy numbers. Fuzzy Sets and Systems, 13 (1984), 1-10.

Received June 4, 1992 